\title{
Ramp Lesions
}

\section{An Unrecognized Posteromedial Instability?}

\author{
Bertrand Sonnery-Cottet, $\mathrm{MD}^{\mathrm{a}, *}$, Raphael Serra Cruz, MD ${ }^{\mathrm{b}, \mathrm{c}, \mathrm{d}}$, \\ Thais Dutra Vieira, $\mathrm{MD}^{\mathrm{a}}$, Rodrigo A. Goes, MD, MSc ${ }^{\mathrm{b}}$, \\ Adnan Saithna, $\mathrm{MD}^{\mathrm{e}, \mathrm{f}}$
}

\section{KEYWORDS}

- Ramp lesion • Hidden lesion • Meniscus • Meniscocapsular tear

- Meniscotibial ligament $\bullet$ Posteromedial corner $\bullet$ Meniscal instability • Meniscal tear

\section{KEY POINTS}

- Meniscal ramp lesions are a "hot topic" because of increasing recognition that they have important biomechanical consequences and also that they occur much more frequently than was previously understood.

- Historically, ramp lesions have been underdiagnosed because of the low sensitivity of MRI and inadequate visualization through standard arthroscopic anterior viewing portals.

- A systematic exploration of the posteromedial compartment of the knee via a trans-notch approach is needed to confirm or refute the presence of a meniscal ramp lesion.

- If left untreated, meniscal ramp lesions may contribute to residual anteroposterior instability in the anterior cruciate ligament-reconstructed knee and may also result in failure of meniscal repair.

- Recent epidemiologic data and definition of risk factors helps to inform an appropriate index of suspicion, identification, and adequate treatment of ramp lesions.

\section{INTRODUCTION}

Meniscal ramp lesions were studied by Hamberg and colleagues ${ }^{1}$ in the 1980 s but at that time were described only as "injuries of the posterior aspect of the medial

Disclosure Statement: B. Sonnery-Cottet receives royalties from, is a paid consultant for, receives research support from, and has made presentations for Arthrex. A. Saithna is a paid consultant for Arthrex Inc.

a Centre Orthopédique Santy, FIFA Medical Centre of Excellence, Groupe Ramsay-Générale de Santé, Hôpital Privé Jean Mermoz, 24 Avenue Paul Santy, Lyon 69008, France; ${ }^{b}$ Instituto Nacional de Traumatologia e Ortopedia, 500 Avendia Brasil, Caju, Rio de Janeiro, 20940-070, Brazil; ' Hospital São Vicente de Paulo, Rio de Janeiro, Rio de Janeiro, Brazil; ${ }^{d}$ Instituto Brasil de Tecnologias da Saúde, Rio de Janeiro, Rio de Janeiro, Brazil; ${ }^{e}$ Sano Orthopedics, 2000 SE Blue Pkwy, Kansas City, MO 64063, USA; ${ }^{f}$ Department of Specialty Medicine (Trauma \& Orthopedic Surgery), Kansas City University, Kansas City, MO, USA

* Corresponding author.

E-mail address: sonnerycottet@aol.com 
meniscus." Later, Strobel ${ }^{2}$ introduced the term ramp lesion and characterized the injury as a longitudinal tear, $2.5 \mathrm{~cm}$ in length, located at the meniscocapsular junction. In 1991, Morgan ${ }^{3}$ described a surgical technique for arthroscopic repair of ramp lesions using a suture hook through a posteromedial portal, and in 2004 Ahn and colleagues ${ }^{4}$ reported a series providing clinical outcomes of repair.

Despite this long history of recognition of ramp lesions, the topic has been infrequently studied over the past few decades, until a recent resurgence in interest. This lack of prior importance attributed to the topic is likely a consequence of an underestimation of their incidence due to a high rate of missed diagnoses, insufficient knowledge about their biomechanical consequences, and an intuitive sense that these lesions could heal spontaneously. The recent interest in these injuries heralds an increasing recognition of their importance and an emerging concept of their association with posteromedial knee instability. The contemporary literature describes these injuries as tears at the posterior meniscocapsular junction and/or tears of the posterior meniscotibial ligament. ${ }^{5-8}$ The expression "hidden lesion," also has been recently used to describe this injury, and the term refers to the difficulty in identifying ramp lesions from standard anterior arthroscopic portals, ${ }^{6,9}$ and also with preoperative MRI, which has low sensititvity. ${ }^{10-12}$

Ramp lesions are hypothesized to occur through a number of possible mechanisms. The most simple is as a result of high forces transmitted through the posteromedial capsule during valgus strain, internal rotation of the tibia, and axial loading at the time of an anterior cruciate ligament (ACL) injury. ${ }^{9}$ The contrecoup injury mechanism, ${ }^{13}$ a compensatory varus alignment and internal rotation of the femur after the initial pivot-shift mechanism, also offers a potential mechanism because it results in impaction between the medial femoral condyle and the medial aspect of the tibial plateau, thus trapping the meniscus. Similarly, Hughston ${ }^{14}$ also suggested these lesions could occur as a result of the meniscus becoming trapped between the femur and tibia, but attributed this to the increased anterior tibial translation that occurs as a result of an ACL injury. Hughston ${ }^{14}$ and others ${ }^{9,13,15}$ have also suggested a potentially important role for the semimembranosus muscle-tendon complex, which in cadaveric study has been found to have a firm attachment to the medial meniscocapsular area in most specimens (86\%). ${ }^{16}$ This close anatomic relationship between the semimembranosus tendon and the meniscocapsular region often can be visualized at the time of ramp repair (Fig. 1). It is hypothesized that contraction of the semimembranosus, secondary to excessive anterior translation of the tibia during an ACL tear or the subsequent contrecoup mechanism may stress this posteromedial area, resulting in a meniscocapsular tear \pm meniscotibial ligament injury and posteromedial instability. ${ }^{14,16}$

The emerging concept of ramp lesions representing a posteromedial instability is based on increasing recognition of the potentially important role of these injuries in knee stability. Ahn and colleagues ${ }^{17}$ and Peltier and colleagues ${ }^{18}$ have demonstrated an increase in anteroposterior instability in ACL-deficient cadaveric knees on creation of ramp lesions and others have also demonstrated significant increases in both internal $^{18}$ and external ${ }^{18,19}$ rotation laxity at all knee flexion angles on creation of ramp lesions \pm meniscotibial ligament injuries. Peltier and colleagues ${ }^{18}$ concluded that these lesions appear to play a significant role in knee stability and also that ramp lesions increase the forces in the ACL. These reports are further supported with the work of numerous other investigators who have demonstrated that isolated ACL reconstruction fails to restore normal joint kinematics and results in residual laxity in the presence of a ramp lesion. ${ }^{4,18,19}$ Furthermore, it has been demonstrated that repair 

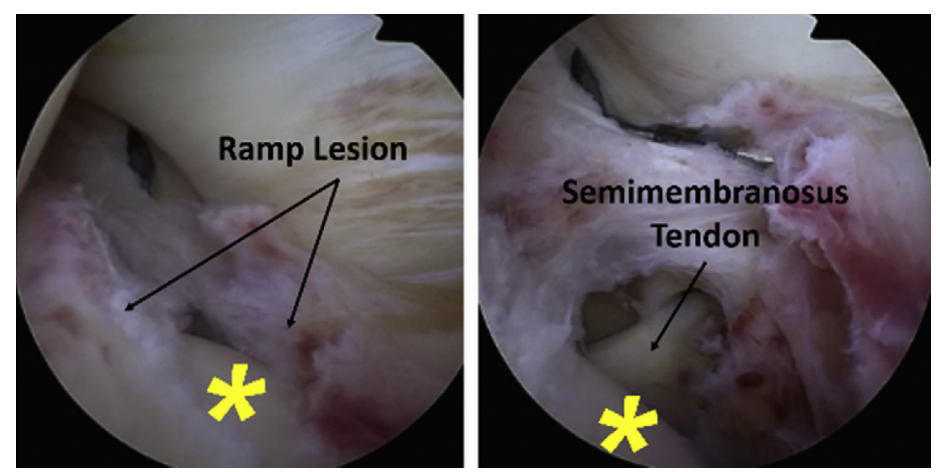

Fig. 1. Arthroscopic trans-notch visualization of the posteromedial compartment of the left knee $(*=$ posteromedial tibial plateau). Retraction of the ramp lesion using an arthroscopy probe placed through the posteromedial portal reveals the close anatomic relationship between the semimembranosus tendon and the meniscocapsular region.

of these lesions abolishes ${ }^{17,19,20}$ the pathologic increase in laxity and therefore provides a biomechanical rationale for identifying and repairing these lesions.

Ramp lesions are reported to occur frequently (9.3\%-24.0\%) in ACL-deficient knees, including in children and adolescents, ${ }^{10,12,17,20,21}$ but Seil and colleagues ${ }^{21}$ identified an even higher rate of $41 \%$ in those with a contact rather than noncontact mechanism of injury. Additional previously reported risk factors for the occurrence of ramp lesions in ACL-deficient knees include male gender, patients younger than 30 years, revision $A C L$ reconstruction, chronic injuries, preoperative side-to-side anteroposterior laxity difference of $6 \mathrm{~mm}$ or more, and the presence of concomitant lateral meniscal tears. ${ }^{22}$ The presence of any of these factors should raise the index of suspicion for the existence of a ramp lesion.

\section{OBJECTIVE DIAGNOSIS}

The accurate detection and treatment of these lesions is essential for restoring knee kinematics and abolishing residual knee laxity. When ramp lesions are overlooked in an $\mathrm{ACL}$ reconstruction, anteroposterior and rotational instabilities persist, ${ }^{14,23,24}$ increasing the risk of failure of the reconstruction. ${ }^{25}$ Because of the high incidence of ramp lesions, surgeons must be highly suspicious of this diagnosis when evaluating a patient with ACL rupture and be aware that there are no specific physical examination tests for ramp lesions. ${ }^{26}$

MRI can be helpful in the detection of a ramp lesion, but it is important to note that it has low to moderate sensitivity and a recognized rate of missed diagnoses. ${ }^{12}$ Recently, a broad range of sensitivities of both $1.5 \mathrm{~T}^{25,27,28}$ and $3 \mathrm{~T}^{27,28} \mathrm{MRI}$ for the detection of ramp lesions has been reported by DePhillipo and colleagues ${ }^{27}(48 \%)$, Hatayama and colleagues ${ }^{28}$ (71.7\%), and Arner and colleagues ${ }^{25}$ (53.9\%-84.6\%), with a high specificity (>90\%). ${ }^{25,28}$ The most specific sign in the MRI evaluation of ramp lesions is the hyperintense signal that can be observed between the meniscus and the capsule ${ }^{29}$ (Fig. 2). However, most acute knee MRI evaluations are performed with the knee in full extension, which reduces the meniscocapsular gap, and can lead to false-negative tests. ${ }^{29}$ The MRI detected presence of bone bruising in the postero-medial tibial plateau has also been associated with ramp lesions, at a rate 

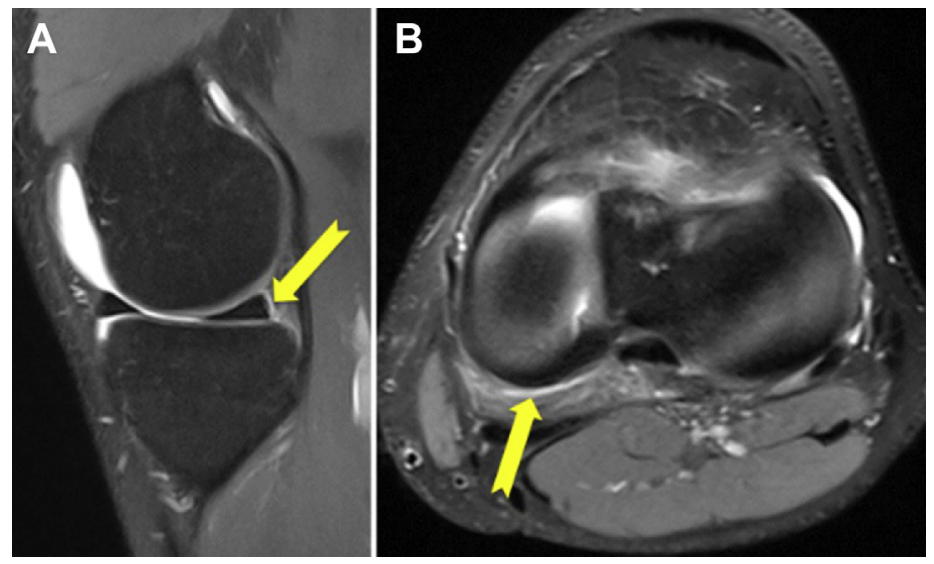

Fig. 2. T2-weighted MRI. (A) Sagittal section showing a hyperintense signal in the posterior meniscocapsular junction of the medial meniscus (arrow). (B) Axial section showing the same lesion (arrow). Observe the difficulty in establishing this diagnosis only by conventional imaging.

that varies from $38.5 \%$ for Hatayama and colleagues, ${ }^{28} 66.3 \%$ for Kumar and colleagues, ${ }^{30}$ and $72.0 \%$ for DePhillipo and colleagues. ${ }^{27}$

Arthroscopy is considered gold standard for diagnosis of ramp lesions. ${ }^{6}$ However, it is not without pitfalls. ${ }^{28}$ Forty percent of ramp lesions are not identified through standard anterior portal visualization and inspection of the posterior compartment via a trans-notch view, and posteromedial probing is required to identify them. ${ }^{6,7}$ This is of particular importance, because these missed tears are repairable. , $^{6,31}$

\section{CLASSIFICATION}

Ramp lesions may be classified into 5 types according to their morphology ${ }^{7}$ (Fig. 3):

Type 1: Meniscocapsular lesions. These lesions are very peripherally located in the synovial sheath. Mobility at probing is very low.

Type 2: Partial superior lesions. These lesions are stable and can be diagnosed only by a trans-notch approach. Mobility at probing is low.

Type 3: Partial inferior or hidden lesions. The lesions are typically subtle or not immediately visible even with trans-notch visualization but can be strongly suggested by significant mobility on probing and also by identification of abnormal tissue quality on needling. ${ }^{5}$

Type 4: A complete tear of the red-red zone. Mobility at probing is very high.

Type 5: A double tear involving the meniscocapsular junction and a second more anterior tear of the posterior horn.

\section{TREATMENT OPTIONS}

As these lesions occur, by definition, in a well-vascularized zone, isolated tears that are small (less than $10 \mathrm{~mm}$ ) and stable may amenable to conservative treatment. $^{32}$ If these conditions are not met, suture of the tear is recommended. One of the most popular surgical techniques for treating meniscal ramp lesions is the use of a posteromedial portal suture hook device. This is used to pass a suture through the injured 

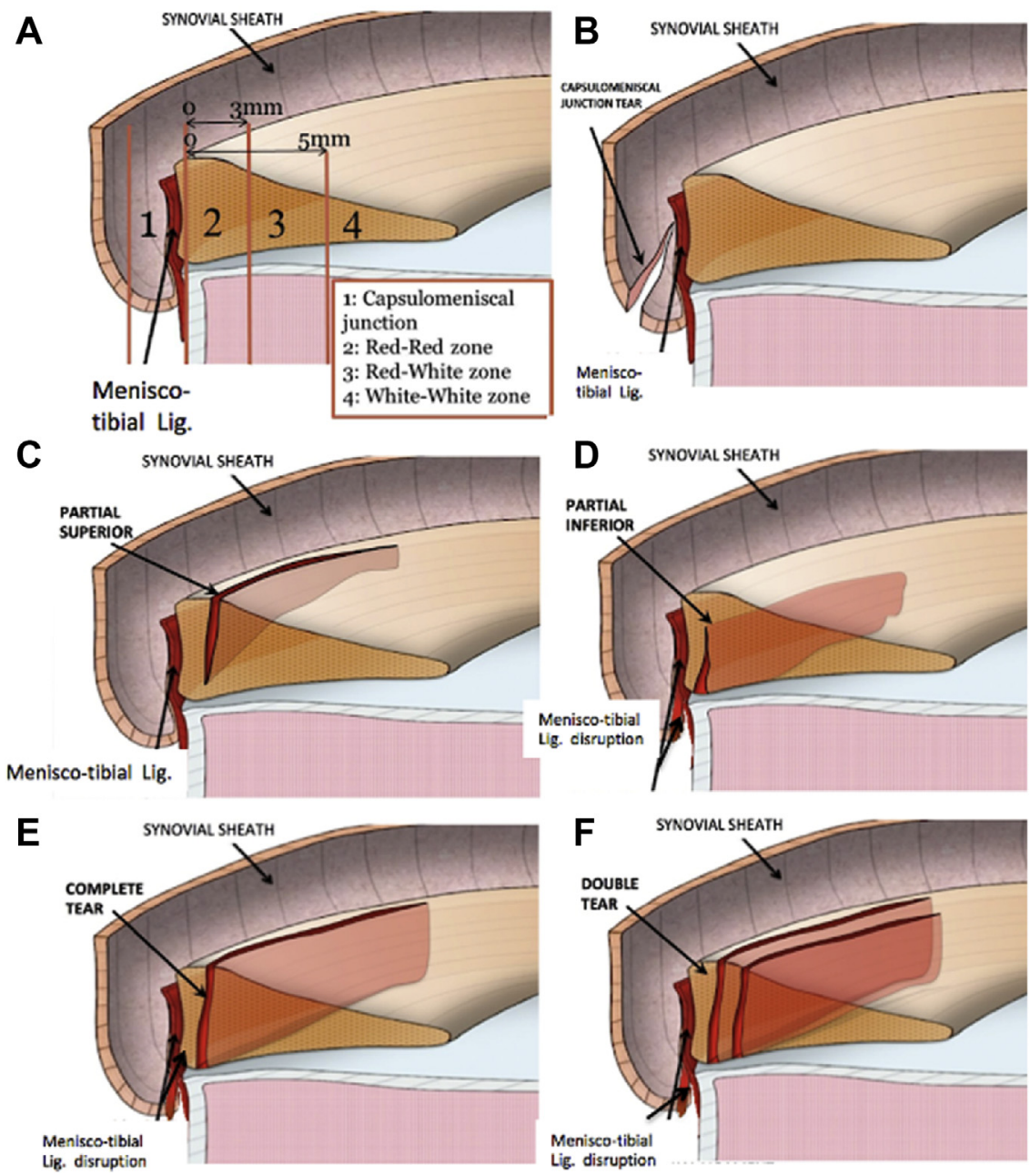

Fig. 3. Ramp lesion classification as proposed by Thaunat and colleagues. ${ }^{7}(A)$ Illustration demonstrating the posterior meniscus-capsular region and the areas of meniscus vascularization. (B) Type 1: Meniscocapsular lesion, located in the synovial sheath. (C) Type 2: Upper partial lesion. $(D)$ Type 3: Lower lesion ("hidden lesion"). (E) Type 4: Complete injury in the red-red area. $(F)$ Type 5: Double tear. Lig., ligament. (From Thaunat M, Fayard JM, Guimaraes TM, Jan N, Murphy CG, Sonnery-Cottet B. Classification and Surgical Repair of Ramp Lesions of the Medial Meniscus. Arthroscopy techniques. Aug 2016;5(4):e871-e875; with permission.)

area, which is then tied with sliding knots (described later in this article). More recently, some investigators have proposed alternative treatments like a classic all-inside suture technique ${ }^{33}$ or even abrasion and trephination of stable lesions (without repair), when they measure less than $1.5 \mathrm{~cm}$, at the time an $\mathrm{ACL}$ reconstruction. ${ }^{32}$ Although these studies provide important information, they do not provide sufficient evidence to guide optimal treatment. In contrast, some surgeons have suggested that acute repair is necessary for ramp lesions because the capsular portion of the torn meniscus has a tendency to retract inferiorly, away from the tibial plateau, making it less likely for 
the tissue to heal spontaneously, thus requiring a specific posteromedial approach for the repair. 4

\section{ARTHROSCOPIC ASSESSMENT}

An important characteristic of the ramp lesion and one of the reasons why it has been underdiagnosed over the years is the difficulty in observing the tear via classic anterior portals, because the medial femoral condyle is located between the arthroscope and the posterior meniscocapsular junction, where it occurs. ${ }^{6}$ This is particularly true in varus knees and in those knees with a tight medial compartment. Some strategies have been proposed to improve visualization of the posteromedial aspect of the knee, including the use of a leg holder for joint distraction along with a large inflow cannula ${ }^{34}$; or pie crusting of the medial collateral ligament. ${ }^{35}$ Despite application of these techniques, the view of the peripheral area of the meniscus remains restricted. ${ }^{6}$ To better assess this region, a trans-notch approach is recommended because it provides better visualization of the posterior meniscocapsular junction. ${ }^{6,7}$ Although a ramp lesion may be suspected by increased mobility of the meniscus, it is essential to perform a direct visualization of the posterior meniscocapsular junction, because some of these tears (types I and II) may seem stable when inspected through anterior portals, even after probing. ${ }^{7}$ Observing this scenario, Sonnery-Cottet and colleagues ${ }^{6}$ have proposed a systematic arthroscopic exploration of the knee joint, ${ }^{6,7}$ using a $30^{\circ}$ scope, which includes 4 steps:

Step 1: Standard arthroscopic exploration

Step 2: Exploration of posteromedial compartment and probing the meniscocapsular junction with a needle

Step 3: Creation of a posteromedial portal

Step 4: Meniscal repair procedure

\section{Step 1: Standard Arthroscopic Exploration}

The patient is positioned supine on the operating table with a tourniquet applied high on the thigh. A foot support is used to maintain the knee at $90^{\circ}$ of flexion during the procedure, while allowing it to be manipulated through full range of motion, as needed (Fig. 4). A standard high lateral parapatellar portal is created for visualization with the arthroscope, while a medial parapatellar portal is created for instrumentation.

The presence of a meniscal tear is then evaluated by meticulous probing of the meniscal tissue. The mobility of the meniscus at probing may lead the surgeon to suspect the presence of a posterior tear even if it is not visible by standard anterior viewing, because ramp lesions classified as types III, IV, and V may be highly mobile when pulled.

\section{Step 2: Exploration of Posteromedial Compartment and Probing the Meniscocapsular Junction with a Needle}

Trans-notch visualization of the posteromedial compartment is performed. The arthroscope is introduced in the anterolateral portal with the knee positioned at $90^{\circ}$ of flexion and then advanced through a triangle limited by the medial femoral condyle, the posterior cruciate ligament, and the tibial spines (Fig. 5A). To facilitate the passage through this space, a valgus force is applied (Fig. 5B) first in extension and then in flexion. If passage remains difficult, the use of a blunt trocar may be helpful. Tibial internal rotation may improve visualization of the tear by causing posterior tibial plateau subluxation and a posterior translation of the medial segment. With this maneuver, 


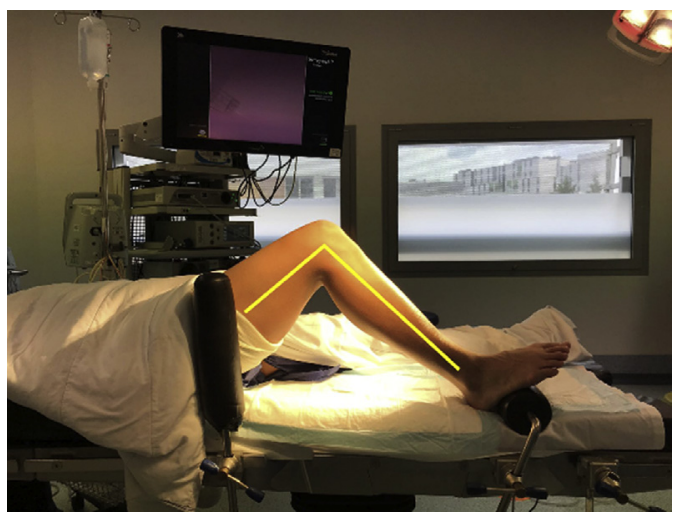

Fig. 4. Positioning of the patient on the operating table, with a support under the ipsilateral foot, sustaining the knee at $90^{\circ}$ of flexion during the procedure while allowing it to be manipulated as needed.

two-thirds of the peripheral lesions from the posterior segment up to the middle segment can be seen (Fig. $5 \mathrm{C}$ ). ${ }^{36}$ In this position, the $30^{\circ}$ optical lens may be adjusted for optimal visualization of the meniscocapsular junction to assess the presence of a ramp lesion without the need for a $70^{\circ}$ scope. $^{7}$ The posterior horn of the medial meniscus can then be explored with a needle to detect a ramp lesion. With the arthroscope positioned in the trans-notch view, transillumination allows the surgeon to observe the nerves and veins that must be avoided. The knee is positioned at $90^{\circ}$ of flexion to minimize the risk of injury to neurovascular structures and a needle is introduced above the hamstring tendons, $1 \mathrm{~cm}$ posterior to the medial tibiofemoral joint line, pointing toward the lesion (Fig. 6). A hidden lesion (type 3) may be found by dissecting the synovial tissue with the needle over the tear. This type of lesion may be suspected, by holding an appropriate index of suspicion based on the presence of risk factors and also if the surgeon notes high mobility of the meniscus at probing during the standard anterior portal arthroscopic evaluation.

\section{Step 3: Creation of a Posteromedial Portal}

After arthroscopically checking the adequate placement of the guiding needle via trans-notch view (Fig. 7A), the needle is removed and a No. 11 scalpel is then used
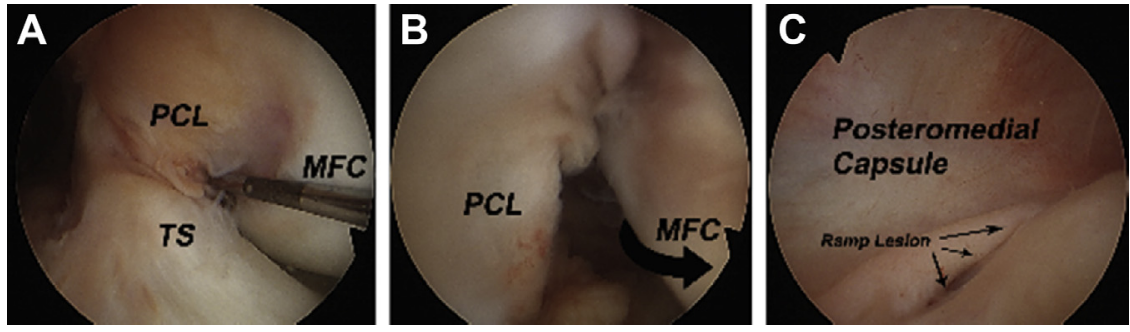

Fig. 5. Arthroscopic view with a $30^{\circ}$ scope of a right knee through the anterolateral portal. (A) Probe is inserted through the anteromedial portal in the space between the posterior cruciate ligament (PCL) and the medial femoral condyle (MFC). (B) Passage of the arthroscope through the space between the PCL and the MFC after a valgus force is applied. (C) Image obtained via trans-notch visualization of the posteromedial region of the knee, observing the posteromedial capsule and the meniscal ramp lesion. TS, tibial spine. 


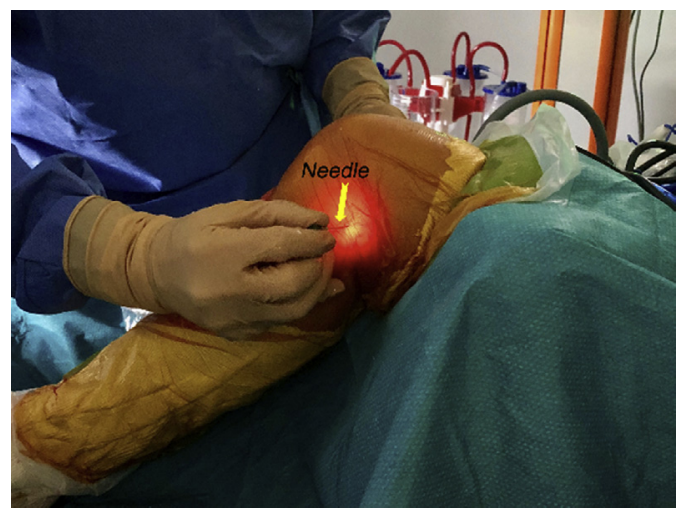

Fig. 6. External image of the knee demonstrating how transillumination with the arthroscope positioned in the trans-notch view helps avoiding neurovascular structures during the creation of the posteromedial portal.

to make a small incision over the posteromedial skin and capsule (Fig. 7B), creating the posteromedial portal. There is no need for a cannula.

\section{Step 4: Meniscal Repair Procedure}

We describe a technique for repair of ramp lesions by the placement of all-inside sutures with the aid of a curved suture hook device (Fig. 8), introduced through the posteromedial portal. A left curved hook device is used for a right knee and viceversa. After correct identification of the tear and debridement of its edges with a shaver (Fig. 9A) or a meniscal rasp, the curved hook device loaded with a No. 0 absorbable monofilament suture (polydioxanone) is introduced through the posteromedial portal (Fig. 9B). It is then manipulated to make the sharp tip penetrate the peripheral wall of the medial meniscus from outside to inside (Fig. 9C) and then penetrate the inner portion of the tear so that both parts are held together (Fig. 9D). The suture is then progressed through the device. A grasper is used to retrieve the free end of the suture via the posteromedial portal (Fig. 9E). A sliding knot is tied with the aid of a knot pusher (Fig. 9F) and then cut. It is the preference of the authors to place sutures at a distance of $10 \mathrm{~mm}$ apart, beginning at the most posterior aspect of the tear to the most anterior one (Fig 10). Internal rotation of the foot helps keep the medial femoral
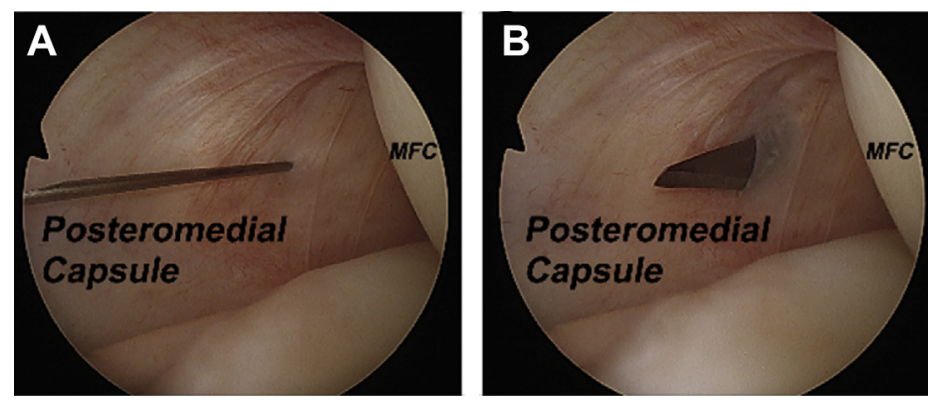

Fig. 7. Arthroscopic image through trans-notch vision showing 2 steps for the creation of the posteromedial portal. $(A)$ Insertion of the needle to verify the correct positioning of the portal and $(B)$ creation of the posteromedial portal with a no. 11 scalpel. MFC, medial femoral condyle. 


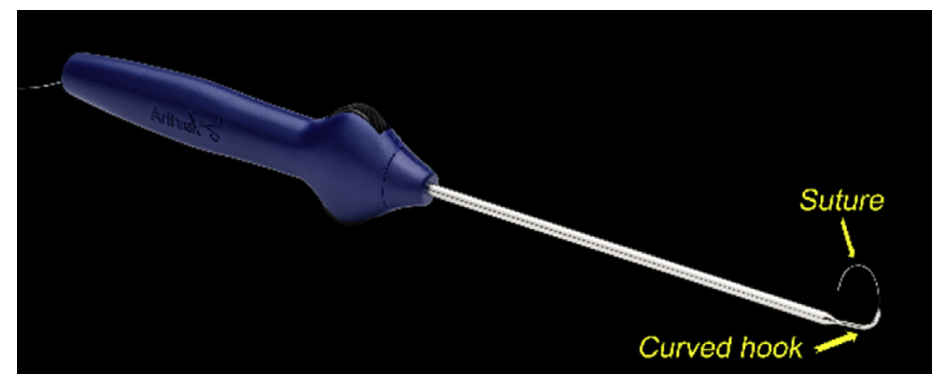

Fig. 8. Image of a curved hook device, with the tip curvature pointing to the left side (ideal for suturing meniscal ramp lesion on a right knee).

condyle away from the posterior segment of the meniscus and facilitates the procedure. After an adequate number of sutures are placed, stabilization of the ramp lesion is tested by probing, with the knee positioned in extension and valgus. If the tear extends anteriorly through the posterior horn and/or body of the meniscus, it should be repaired with the surgeon's preferred technique.

\section{REHABILITATION PROTOCOL}

There is no consensus in the literature regarding rehabilitation following ramp repair. Most investigators choose to use their standard ACL rehabilitation protocol. $6,10,31,37$ In the case of isolated ramp lesions, a standard meniscal repair rehabilitation protocol should be followed. ${ }^{31}$ Most protocols agree that early knee motion is beneficial; however, knee hyperflexion is associated with anterior tibial translation, which can increase the stress on the repair. ${ }^{12}$ In our practice, the postoperative management of an ACL

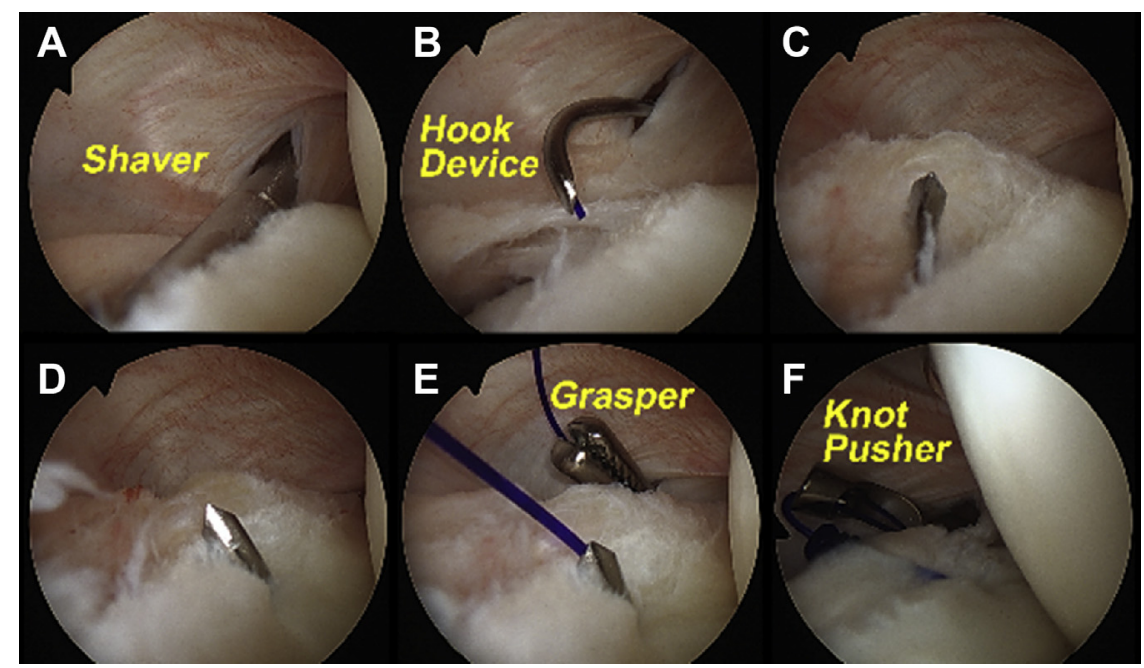

Fig. 9. Detailing of a meniscal ramp suture procedure. $(A)$ Revitalization of the edges of the lesion using a shaver. $(B)$ Suture device inserted through the posteromedial portal. (C) Penetration of the peripheral edge of the lesion. (D) Penetration of the suture device through the inner portion of the tear, encompassing both ends of the lesion. (E) Progression of the suture, which is then captured out of the knee with the aid of a grasper. $(F)$ First stitch being completed using a knot pusher. 


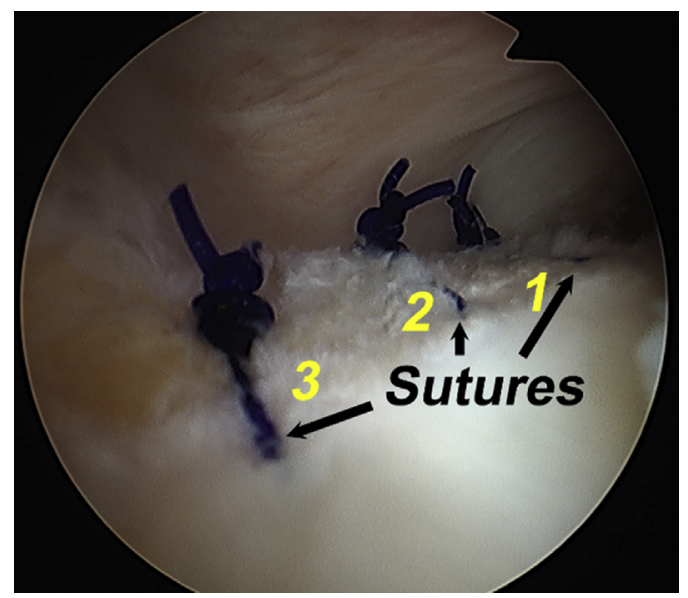

Fig. 10. Final arthroscopic aspect of a meniscal ramp repair, as seen by trans-notch view, in which 3 stitches were required to close the lesion. Numbers represent the sequence of suturing.

reconstruction associated with ramp repair is modified by restriction of the active and passive range of motion to 0 to $90^{\circ}$ of flexion during the first 6 weeks. Progression to full weight bearing is allowed by postoperative week 3 . Jogging is permitted after week 12 to 16 , pivot activity at 6 months, and full activity at 9 months for all patients. ${ }^{36}$

\section{OUTCOMES}

Although several investigators have reported clinical outcomes of repair of ramp lesions, the number of publications is fairly limited. ${ }^{4,10,22,36,38}$ Jan and colleagues ${ }^{39}$ reported that arthroscopic suture hook repair via a posteromedial portal provides good healing rates without an increase in morbidity due to the supplementary portal. Reported failure rates range from $2.6 \%{ }^{4}$ to $12.0 \%,{ }^{38}$ with $30 \%$ of failures occurring between $2^{17}$ and 4 years after the index procedure. ${ }^{22,40}$ Typically the new lesion involves a smaller and more central part of the meniscus, anterior to the previously passed sutures. Often there is the appearance of a "postage-stamp" effect, possibly implicating the suture hook and/or presence of nonabsorbable sutures in the etiology of the new lesion. ${ }^{39}$ However, this pattern leads to a smaller secondary resection than had partial meniscectomy been performed initially. ${ }^{41}$

It is a well-recognized phenomenon that there is an increased failure rate of meniscal repairs in ACL-deficient knees due to abnormal knee kinematics and excessive loads on the repaired lesion. ${ }^{40,42}$ It is therefore unsurprising to note that the secondary meniscectomy rate is significantly reduced (twofold) in those patients who have undergone a combined ACLR and anterolateral ligament reconstruction (ALLR) when compared with those undergoing isolated ACLR. This is because isolated ACLR fails to restore normal knee kinematics in those knees in which a combined ACL and anterolateral injury exists. ${ }^{43}$ These findings support the results of previous study that has demonstrated the protective effect of ALLR on medial meniscal repairs. ${ }^{22,38}$ In addition, it is valuable to recognize the possibility that the important biomechanical consequences of these injuries may risk a greater failure rate of ACL grafts in knees with unrepaired ramp lesions. ${ }^{37}$

\section{SUMMARY}

Meniscal ramp lesions are common but frequently underrecognized in the ACL-injured knee. It is important to highlight that both preoperative MRI and arthroscopic 
evaluation via classic anterior portals are associated with high rates of missed diagnoses. For that reason, a systematic exploration of the posteromedial compartment is advocated in all ACL-injured knees. Failure to recognize and repair ramp lesions is associated with persistent anterior and rotational knee laxity, suggesting it makes part of a posteromedial instability, in the setting of an ACL tear. Concomitant suture repair of these lesions, via a posteromedial portal, can restore normal biomechanics and is associated with excellent clinical outcomes.

\section{AUTHORS' OPINION}

Interesting fact: All over the world, in orthopedic meetings, the meniscal root lesion is more extensively debated than the ramp lesion. This is despite the fact that ramp lesions are a lot more common (24\% in ACLR in our experience). This greater focus on root tears is perhaps because of the well-recognized loss of hoop force distribution and profound biomechanical consequences of root tears. However, it is increasingly recognized that ramp lesions also have important biomechanical consequences including persistent anteroposterior (AP) and rotational laxity and an association with higher ACL graft failure rates. It is the authors' opinion that ramp lesions require further study and greater attention because of their underrecognized role in posteromedial knee instability.

Changing patterns: The authors have observed a decrease in the failure rate for all types of medial meniscus suture repair since 2012 , from $25 \%$ to $7 \%$, with a mean follow-up of 5 years. This observation is attributed to the systematic exploration of the posteromedial compartment, allowing us to discover and repair these previously underrecognized ramp lesions. It is our opinion that the lower rate of failure of all types of medial meniscus repair is a function of improved knee kinematics, through abolishing the greater AP and rotational laxity associated with ramp lesions and also due to the better quality of repair as a result of improved lesion access and debridement via a posteromedial portal.

Technical Tip: When evaluating the posteromedial compartment, it is important to avoid missed diagnoses of hidden lesions. There are tears in which the superior surface often appears subtlety abnormal but the tear is not apparent unless it is probed with a needle and the intact superficial fibers are separated revealing the ramp lesion.

\section{REFERENCES}

1. Hamberg P, Gillquist J, Lysholm J. Suture of new and old peripheral meniscus tears. J Bone Joint Surg Am 1983;65(2):193-7.

2. Strobel MJ. Menisci. Manual of arthroscopic surgery. New York: Springer; 1988. p. 171-8.

3. Morgan CD. The "all-inside" meniscus repair. Arthroscopy 1991;7(1):120-5.

4. Ahn JH, Kim SH, Yoo JC, et al. All-inside suture technique using two posteromedial portals in a medial meniscus posterior horn tear. Arthroscopy 2004;20(1): 101-8.

5. Smigielski R, Becker R, Zdanowicz U, et al. Medial meniscus anatomy-from basic science to treatment. Knee Surg Sports Traumatol Arthrosc 2015;23(1):8-14.

6. Sonnery-Cottet B, Conteduca J, Thaunat M, et al. Hidden lesions of the posterior horn of the medial meniscus: a systematic arthroscopic exploration of the concealed portion of the knee. Am J Sports Med 2014;42(4):921-6.

7. Thaunat M, Fayard JM, Guimaraes TM, et al. Classification and surgical repair of ramp lesions of the medial meniscus. Arthrosc Tech 2016;5(4):e871-5. 
8. Pedersen RR. The medial and posteromedial ligamentous and capsular structures of the knee: review of anatomy and relevant imaging findings. Semin Musculoskelet Radiol 2016;20(1):12-25.

9. Laprade RF. The Menisci-a comprehensive review of their anatomy, biomechanical function and surgical treatment. Springer-Verlag Berlin Heidelberg; 2017.

10. Liu $X$, Feng $H$, Zhang $H$, et al. Arthroscopic prevalence of ramp lesion in 868 patients with anterior cruciate ligament injury. Am J Sports Med 2011;39(4): 832-7.

11. Seil R, VanGiffen N, Pape D. Thirty years of arthroscopic meniscal suture: what's left to be done? Orthop Traumatol Surg Res 2009;95(8 Suppl 1):S85-96.

12. Bollen SR. Posteromedial meniscocapsular injury associated with rupture of the anterior cruciate ligament: a previously unrecognised association. J Bone Joint Surg $\mathrm{Br}$ 2010;92(2):222-3.

13. Yoon $\mathrm{KH}$, Yoo JH, Kim KI. Bone contusion and associated meniscal and medial collateral ligament injury in patients with anterior cruciate ligament rupture. J Bone Joint Surg Am 2011;93(16):1510-8.

14. Hughston JC. Knee ligaments: injury and repair. Saint Louis (MO): Mosby; 1993.

15. Smith JP 3rd, Barrett GR. Medial and lateral meniscal tear patterns in anterior cruciate ligament-deficient knees. A prospective analysis of 575 tears. Am J Sports Med 2001;29(4):415-9.

16. DePhillipo NN, Moatshe G, Chahla J, et al. Quantitative and qualitative assessment of the posterior medial meniscus anatomy: defining meniscal ramp lesions. Am J Sports Med 2019;47(2):372-8.

17. Ahn JH, Bae TS, Kang KS, et al. Longitudinal tear of the medial meniscus posterior horn in the anterior cruciate ligament-deficient knee significantly influences anterior stability. Am J Sports Med 2011;39(10):2187-93.

18. Peltier A, Lording T, Maubisson L, et al. The role of the meniscotibial ligament in posteromedial rotational knee stability. Knee Surg Sports Traumatol Arthrosc 2015;23(10):2967-73.

19. Stephen JM, Halewood C, Kittl C, et al. Posteromedial meniscocapsular lesions increase tibiofemoral joint laxity with anterior cruciate ligament deficiency, and their repair reduces laxity. Am J Sports Med 2016;44(2):400-8.

20. Reider B. Ramped up. Am J Sports Med 2017;45(5):1001-3.

21. Seil R, Mouton C, Coquay J, et al. Ramp lesions associated with ACL injuries are more likely to be present in contact injuries and complete ACL tears. Knee Surg Sports Traumatol Arthrosc 2018;26(4):1080-5.

22. Sonnery-Cottet B, Praz C, Rosenstiel N, et al. Epidemiological evaluation of meniscal ramp lesions in 3214 anterior cruciate ligament-injured knees from the SANTI Study Group Database: a risk factor analysis and study of secondary meniscectomy rates following 769 ramp repairs. Am J Sports Med 2018;46(13): 3189-97.

23. Noyes FR, Chen RC, Barber-Westin SD, et al. Greater than 10-year results of redwhite longitudinal meniscal repairs in patients 20 years of age or younger. Am J Sports Med 2011;39(5):1008-17.

24. Mariani PP. Posterior horn instability of the medial meniscus a sign of posterior meniscotibial ligament insufficiency. Knee Surg Sports Traumatol Arthrosc 2011;19(7):1148-53.

25. Arner JW, Herbst E, Burnham JM, et al. MRI can accurately detect meniscal ramp lesions of the knee. Knee Surg Sports Traumatol Arthrosc 2017;25(12):3955-60.

26. Bronstein RD, Schaffer JC. Physical examination of the knee: meniscus, cartilage, and patellofemoral conditions. J Am Acad Orthop Surg 2017;25(5):365-74. 
27. DePhillipo NN, Cinque ME, Chahla J, et al. Incidence and detection of meniscal ramp lesions on magnetic resonance imaging in patients with anterior cruciate ligament reconstruction. Am J Sports Med 2017;45(10):2233-7.

28. Hatayama K, Terauchi M, Saito K, et al. Magnetic resonance imaging diagnosis of medial meniscal ramp lesions in patients with anterior cruciate ligament injuries. Arthroscopy 2018;34(5):1631-7.

29. Hash TW 2nd. Magnetic resonance imaging of the knee. Sports Health 2013;5(1): 78-107.

30. Kumar NS, Spencer T, Cote MP, et al. Is edema at the posterior medial tibial plateau indicative of a ramp lesion? an examination of 307 patients with anterior cruciate ligament reconstruction and medial meniscal tears. Orthop J Sports Med 2018;6(6). 2325967118780089.

31. Chahla J, Dean CS, Moatshe G, et al. Meniscal ramp lesions: anatomy, incidence, diagnosis, and treatment. Orthop J Sports Med 2016;4(7). 2325967116657815.

32. Liu $X$, Feng $H$, Hong $L$, et al. A prospective randomized control trial of arthroscopic surgery for stable ramp lesion of the medial meniscus. Zhonghua Wai Ke Za Zhi 2017;55(3):161-5 [in Chinese].

33. Chen Z, Li WP, Yang R, et al. Meniscal ramp lesion repair using the FasT-fix technique: evaluating healing and patient outcomes with second-look arthroscopy. J Knee Surg 2018;31(8):710-5.

34. Carson WG Jr. Arthroscopic techniques to improve access to posterior meniscal lesions. Clin Sports Med 1990;9(3):619-32.

35. Fakioglu O, Ozsoy MH, Ozdemir HM, et al. Percutaneous medial collateral ligament release in arthroscopic medial meniscectomy in tight knees. Knee Surg Sports Traumatol Arthrosc 2013;21(7):1540-5.

36. Thaunat M, Jan N, Fayard JM, et al. Repair of meniscal ramp lesions through a posteromedial portal during anterior cruciate ligament reconstruction: outcome study with a minimum 2-year follow-up. Arthroscopy 2016;32(11):2269-77.

37. Li WP, Chen Z, Song B, et al. The FasT-fix repair technique for ramp lesion of the medial meniscus. Case Rep Orthop 2015;27(1):56-60.

38. Sonnery-Cottet B, Saithna A, Blakeney WG, et al. Anterolateral ligament reconstruction protects the repaired medial meniscus: a comparative study of 383 anterior cruciate ligament reconstructions from the SANTI Study Group with a minimum follow-up of 2 years. Am J Sports Med 2018;46(8):1819-26.

39. Jan N, Sonnery-Cottet B, Fayard JM, et al. Complications in posteromedial arthroscopic suture of the medial meniscus. Orthop Traumatol Surg Res 2016;102(8s): S287-93.

40. Rochecongar G, Plaweski S, Azar M, et al. Management of combined anterior or posterior cruciate ligament and posterolateral corner injuries: a systematic review. Orthop Traumatol Surg Res 2014;100(8 Suppl):S371-8.

41. Pujol N, Beaufils P. Healing results of meniscal tears left in situ during anterior cruciate ligament reconstruction: a review of clinical studies. Knee Surg Sports Traumatol Arthrosc 2009;17(4):396-401.

42. Duchman KR, Westermann RW, Spindler KP, et al. The fate of meniscus tears left in situ at the time of anterior cruciate ligament reconstruction: a 6-year follow-up study from the MOON Cohort. Am J Sports Med 2015;43(11):2688-95.

43. Inderhaug E, Stephen JM, Williams A, et al. Anterolateral tenodesis or anterolateral ligament complex reconstruction: effect of flexion angle at graft fixation when combined with ACL reconstruction. Am J Sports Med 2017;45(13):3089-97. 